\title{
EFFECTS OF SONICATION ON SIZE DISTRIBUTION AND ENTRAPMENT OF LYNESTRENOL TRANSFEROSOME
}

\author{
ISKANDARSYAH ISKANDARSYAH*, CAMELIA DWI PUTRI MASRIJAL, HARMITA HARMITA
}

Department of Pharmacy, Physical Pharmacy Laboratory, Faculty of Pharmacy, Universitas Indonesia, Indonesia. Email: aya_2803@yahoo.com Received: 26 September 2019, Revised and Accepted: 17 December 2019

ABSTRACT

Objective: The aim of this study was to develop transferosome vesicles for the transdermal drug delivery of lynestrenol.

Methods: The lynestrenol transferosome vesicle was made by encapsulating the drug in a variation of phosphatidylcholine and Tween 80 by the thinlayer hydration method. The resulting transferosome vesicles were modified with a time variation of 30, 60, 90, and 120 min, and sonication variations were paused and not paused. Particle size evaluation, polydispersity (PDI), and entrapment efficiency (\%EE) were carried out on the variation of sonication time.

Results: The evaluation results showed that sonication without pauses showed better \%EE and particle size than sonication with pauses and increasing concentration of Tween 80 (edge activator). The \%EE increased, and particle size decreased with increasing sonication time; PDI of vesicles was heterogeneous with increasing sonication time. The \%EE in formulas F1 and F2 after 120 min was $73.06 \%$ and $76.06 \%$ (paused) and $80.40 \%$ and $82.97 \%$ (without paused). The particle size of formula F1 and F2 after $120 \mathrm{~min} 575.4 \mathrm{~nm}$ and $471.6 \mathrm{~nm}$ (paused) and $524.1 \mathrm{~nm}$ and $434.7 \mathrm{~nm}$ (without paused). The PDI formulas of F1 and F2 after 120 min were 0.69 and 0.763 (paused) and 0.84 and 0.59 (without paused).

Conclusion: Based on the results of the transferosome vesicle characteristics, it was shown that the optimal vesicle composition for packaging lynestrenol was vesicles that were composed of phosphatidylcholine and Tween 80 without pauses and could potentially be used as a transdermal drug delivery system.

Keywords: Transferosome, Transdermal drug delivery system, Sonication, Lynestrenol.

(C) 2020 The Authors. Published by Innovare Academic Sciences Pvt Ltd. This is an open access article under the CC BY license (http://creativecommons. org/licenses/by/4. 0/) DOI: http://dx.doi.org/10.22159/ijap.2020.v12s1.FF053

\section{INTRODUCTION}

The stratum corneum, the outermost barrier of the skin which provides protection against the entry of foreign materials, imposes an obstacle to the transdermal diffusion of drugs [1]. Since only a few smallmolecule drugs with high lipophilicity can naturally permeate through the hydrophobic stratum corneum, a vast majority of hydrophilic drugs have been ruled out against transdermal delivery. Even after the drug successfully penetrates the stratum corneum, it may be degraded prematurely by epidermal enzymes, resulting in decreased bioavailability. Latency in the onset of action as well as a lack of pharmacokinetic control may also undermine the reliability of the transdermal delivery. One transdermal formulation that may overcome some of these obstacles is the transferosome [2].

Transferosomes for potential transdermal application contain a mixture of lipids and biocompatible membrane softeners. The optimal mixture leads to flexibility of the elastic liposomal membranes and to the possibility of penetration through channels of the skin which are opened by the carriers. Transferosome is a supramolecular entity that can pass through a permeability barrier and thereby transport material from the application site to the destination site. Transferosome is more elastic than standard liposomes and therefore is used as a novel carrier for effective transdermal drug delivery $[3,4]$.

These nanoparticles have been advocated as one potential delivery system, which can resolve the skin permeation limitation of drugs to a great extent. The likelihood of nanoparticle penetration across the skin has recently been reviewed by the Scientific Committee on Consumer Products in relation to dermal exposure $[4,5]$. The very small particles of less than $100 \mathrm{~nm}$, such as functionalized fullerenes and quantum dots, can penetrate into the viable tissues of a skin. The metallic and quantum dot nanoparticles greater than $200 \mathrm{~nm}$ can also penetrate through the stratum corneum and into the viable epidermis [6,7].

\section{MATERIALS AND METHODS}

\section{Materials}

Lynestrenol was purchased from IPC Pirumadara (India); Tween 80 and Phospholipon ${ }^{\circledR} 90 \mathrm{G}$ (Soya phosphatidylcholine $\geq 91 \%$ ) were purchased from Lipoid (GMBH, Jerman); cholesterol, potassium dihydrogen phosphate monohydrate, sodium hydroxide, and dichloromethane were purchased from Merck (Darmstadt, German). Methanol and acetonitrile were of analytical grade and were purchased from Jakarta, Indonesia.

\section{Methods}

Preparation of transferosomes

The thin-film hydration method was used for the preparation of the vesicles. The lipid Phospholipon ${ }^{\circledR}$ 90G and edge activator Tween 80 were employed for the formulation of the vesicles. For the formulation of vesicles, soya phosphatidylcholine, cholesterol, and Tween 80 at different concentrations were selected as the lipid, membrane stabilizer, and edge activator, respectively. The lipid, edge activator, and drug (lynestrenol) were weighed and then dissolved in dichloromethane. This solution was evaporated on a rotary evaporator (Buchi R-100, Switzerland) at $40^{\circ} \mathrm{C}$ with a vacuum evaporator on 850 mbar (Buchi V-100, Switzerland) until became thin-layer film. Nitrogen gas was allowed to flow through the thin film, and it was then stored in the refrigerator for $24 \mathrm{~h}$. The thin film was then hydrated with $\mathrm{pH} 7.4$ phosphate-buffered saline (PBS) [8,9]. The formulas of the different transferosomes are shown in Table 1.

\section{Sonication of the formulas}

The prepared transferosomes were sonicated with a state of amplitude of 100 , time $10 \mathrm{~min}$, and pulse 3 (amp/time/pulse=100/10/3). 
Transferosomes of the four formulas were sonicated without intervals of $30,60,90$, and $120 \mathrm{~min}$ and sonicated with intervals of 15 min every $30,60,90$, and $120 \mathrm{~min}$. The results of each time are paused and without paused in measuring particle size, zeta potential, polydispersity (PDI), and adsorption efficiency [9].

Particle size, PDI index, and zeta potential measurements

Zeta potential, PDI index, and particle size for each transferosome formula were determined using dynamic light scattering techniques at $25^{\circ} \mathrm{C}$ using the particle size system, (Zetasizer, Malvern, UK; Beckman Coulter, USA). Transferosomal colloidal dispersions were diluted with pure water before measurement [10]

\section{Transferosome entrapment efficiency (\%EE) of lynestrenol}

From the two transferosome formulas each, $1 \mathrm{ml}$ from each was centrifuged at $10,000 \mathrm{rpm}$ for $1 \mathrm{~h}\left(4^{\circ} \mathrm{C}\right)$ to allow the separation of the unentrapped drug. One milliliter of transferosome was added to $1 \mathrm{~mL}$ of Triton X $(0.1 \%)$ and centrifuged at $10,000 \mathrm{rpm}$ for $1 \mathrm{~h}\left(4^{\circ} \mathrm{C}\right)$ to allow the separation of the total drug. After removal of the supernatant, the supernatant was added to methanol and then analyzed by highpressure liquid chromatography (HPLC) at $204 \mathrm{~nm}$ using an HPLC system (Shimadzu, Jepang) [11]. The \%EE of MIC in the prepared transferosome was calculated applying the following equation:

$$
\% \mathrm{EE}=\frac{\text { Total amount of drug }- \text { Amount of entrapped }}{\text { Total amount of drug }} \times 100 \%
$$

\section{RESULTS AND DISCUSSION}

Formula F2 without pausing during sonication resulted in the smallest particle size of $443.90 \mathrm{~nm}$ [Fig. 1]. Sonication crossings were paused and not paused. Based on this, the results indicated that sonication without pausing resulted in a smaller size. This change was due to the energy obtained when sonication was not lost. Sonication with pausing caused

Table 1: The formulas of lynestrenol transferosome

\begin{tabular}{lllll}
\hline Ingredients & \multicolumn{4}{l}{ Concentration (\%) } \\
\cline { 2 - 5 } & F1 & F2 & F3 & F4 \\
\hline Lynestrenol & $300 \mathrm{mg}$ & $300 \mathrm{mg}$ & $300 \mathrm{mg}$ & $300 \mathrm{mg}$ \\
Phospholipon 90G & 9 & 8 & 9 & 10 \\
Tween 80 & 1 & 2 & - & - \\
Cholesterol & - & - & 1 & - \\
PBS, pH 7.4 & Adjust to & Adjust to & Adjust to & Adjust to \\
& $50 \mathrm{~mL}$ & $50 \mathrm{~mL}$ & $50 \mathrm{~mL}$ & $50 \mathrm{~mL}$ \\
\hline
\end{tabular}

the energy to disappear so when the sample is resonicated, it took time to produced energy to reduce the particle size. In formula F1_P and F2_P both F1_WP F2_WP, an increase in the concentration of Tween 80 resulted in a smaller size than the particle size of F2_P $(558.10 \mathrm{~nm})$.

This may be related to the general concept that using surfactants with lower hydrophilic-lipophilic balance (HLB) results in the preparation of smaller sized vesicles. Therefore, transferosome prepared with Tween 80 which is a hydrophilic surfactant, with small concentrations of Tween 80 (F1P) has smaller sizes than those prepared with higher concentrations. The direct proportionality between vesicle size and the HLB of the surfactant can be explained by the reduction in surface free energy resulting from high hydrophobicity. The same explanation applies to decreases in vesicle size with increases in the total lipid content. Formulas F3 and F4 either paused or not paused produced larger sized vesicles than FI and F2. The increased vesicle size of transferosomes made using F3 is because the cholesterol stiffens the transferosome which makes reducing the size more difficult. The lack of Tween 80, which is an edge activator, in the F4 formula results in reduced flexibility of the vesicles in response to sonication and increased vesicle size.

Formulas F3 and F4 either paused or not paused produced a larger size vesicle than either FI or F2; this is because the effect of cholesterol on the structure of the transferosome became stiff so that reducing the size became more difficult. The F4 formula was only composed of phospholipids, so the structure of the transferosome was only the main constituent of the lipid bilayer and there was no edge activatorlike Tween 80 which was flexible so that when reducing the size with sonication was more difficult [12].

As shown in Table 2, all the formulas have negative zeta potentials. Negative values of the zeta potential are mainly due to the presence of ionic nitrogen atoms in the structure of lynestrenol.

The magnitude of the zeta potential in all of the formulations was high enough to provide stability for the vesicles and to prevent coagulation.

The effect of sonication without pauses showed better results in F1_WP and F2_WP formulas ranging from $-23.80 \mathrm{mv}$ to $-18.52 \mathrm{mv}$ (F1_WP) and $-31.36 \mathrm{mv}$ to $-15.98 \mathrm{mv}$. In this formula, the increased concentration of Tween affects the coagulation of the transferosome. The zeta potential for the F3_P formula ranged from $-31.36 \mathrm{mv}$ to $-50.20 \mathrm{mv}$, which is likely due to the presence of cholesterol. The increased zeta potentials of F4 are likely due to the vesicle being composed fully of a phospholipid bilayer [Fig. 2].

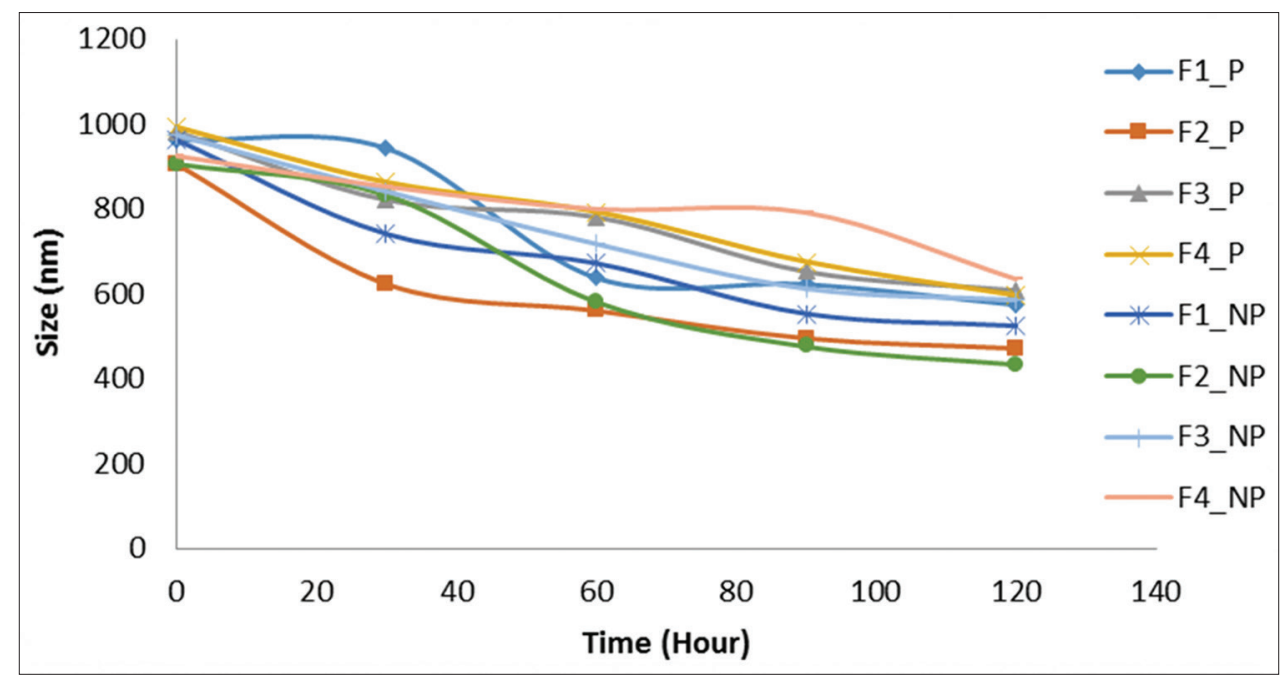

Fig. 1: Particle size of four formulas. Note: F1 (phospholipid:Tween 80 9:1), F2 (phospholipid:Tween 80 8:2), F3 (phospholipid:cholesterol 9:1), F4 (phospholipid:Tween 80 10:0) P: paused; WP: Without paused 
Table 2: Polydispersity and zeta potential of transferosome formulations

\begin{tabular}{|c|c|c|c|c|c|c|c|c|}
\hline \multirow[t]{2}{*}{ Time (h) } & \multicolumn{8}{|c|}{ Polydispersity } \\
\hline & F1_P & F2_P & F3_P & F4_P & F1_WP & F2_WP & F3_WP & F4_WP \\
\hline 30 & 0.655 & 0.603 & 0.494 & 0.838 & 0.816 & 0.441 & 0.494 & 0.838 \\
\hline 90 & 0.598 & 0.598 & 0.672 & 0.878 & 0.677 & 0.513 & 0.443 & 0.710 \\
\hline \multirow[t]{2}{*}{120} & 0.692 & 0.763 & 0.54 & 0.437 & 0.934 & 0.934 & 0.763 & 0.269 \\
\hline & \multicolumn{8}{|c|}{ Zeta potential (mv) } \\
\hline 0 & -23.8 & -21.86 & -31.36 & -40.46 & -23.80 & -21.86 & -31.36 & -40.46 \\
\hline 30 & -21.74 & -20.64 & -44.63 & -40.26 & -22.14 & -19.87 & -44.63 & -40.26 \\
\hline 60 & -20.41 & -18.71 & -49.50 & -49.30 & -20.78 & -16.87 & -42.73 & -49.30 \\
\hline 90 & -18.76 & -18.03 & -39.4 & -46.26 & -19.41 & -16.01 & -39.97 & -39.3 \\
\hline 120 & -17.98 & -16.25 & -50.20 & -42.26 & -18.52 & -15.98 & -47.00 & -40.20 \\
\hline
\end{tabular}

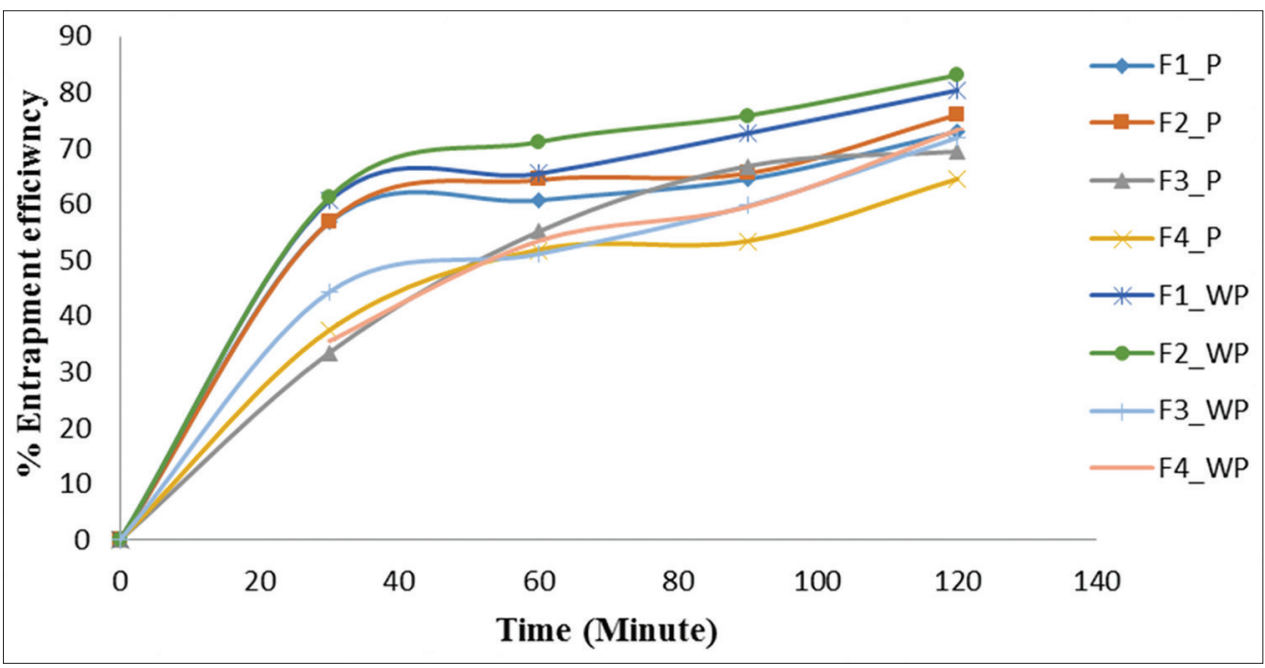

Fig. 2: Transferosome entrapment efficiency. Note: F1 (phospholipid:Tween 80 9:1), F2 (phospholipid:Tween 80 8:2),

F3 (phospholipid:cholesterol 9:1), F4 (phospholipid:Tween 80 10:0), P: Paused; WP: Without paused

\section{CONCLUSION}

Nano-transferosomes of the desired size, zeta potential, and entrapment efficiency were successfully prepared for transdermal lynestrenol delivery. The effect of sonication without pauses gives better results on the nature of transferosome compared to sonication with pauses. The effect of Tween 80 on the transferosome structure could reduce the size, zeta potential, and absorption efficiency. Cholesterol in the lipid bilayer transferosome structure made the structure stiff so that the properties of the vesicles are less desirable compared to those made using Tween 80 .

\section{ACKNOWLEDGMENTS}

The authors gratefully acknowledge to Directorate of Research and Community Engagements of Universitas Indonesia for financial support: Hibah PITTA B number NKB:-0476/UN2.R3.1/HKP.05.00/2019.

\section{CONFLICTS OF INTEREST}

All authors have none to declare.

\section{REFERENCES}

1. Verma P, Ram A, Jha AK, Mishra A, Thakur A. Phosphatidylcholine: A revolution in drug delivery technology. Int J Pharm Sci Res 2010;1:1-2.

2. Jain CP, Vyas SP, Dixit VK. Niosomal system for delivery of rifampicin to lymphatics. Int J Pharma Sci 2006;68:5758.

3. Baroli B, Ennas MG, Loffredo F, Isola M, Pinna R, López-Quintela MA. Penetration of metallic nanoparticles in human full-thickness skin. J
Invest Dermatol 2007;127:1701-12.

4. Prow TW, Grice JE, Lin LL, Faye R, Butler M, Becker W, et al. Nanoparticles and microparticles for skin drug delivery. Adv Drug Deliv Rev 2011;63:470-91

5. Ryman-Rasmussen JP, Riviere JE, Monteiro-Riviere NA. Variables influencing interactions of untargeted quantum dot nanoparticles with skin cells and identification of biochemical modulators. Nano Lett 2007;7:1344-8.

6. Benson HA. Transfersomes for transdermal drug delivery. Expert Opin Drug Deliv 2006:3:727-37.

7. Cevc G, Blume G. New, highly efficient formulation of diclofenac for the topical, transdermal administration in ultradeformable drug carriers, transfersomes. Biochim Biophys Acta 2001;1514:191-205.

8. Iskandarsyah I, Alvina WP, Ernysagita E. Penetration test of caffein in ethosome and desmosome gel using an in vitro method. Int J Appl Pharm 2017;9:1.

9. Anggraini W, Erny S, Iskandarsyah I. Effect of hifrophilicity surfactant toward characterization and in vitro transfersomes penetration in gel using Franz diffusion test. Int J Appl Pharm 2017;9:1

10. Reza MI, Divya G, Rahul KG, Musarrat HW. Formulation of ketoconazole loaded nano dispersive gel using swollen micelles technique and its in vitro characterization. Int J Pharm Pharm Sci 2018;10:162-6.

11. Nurleli N, Iskandarsyah I, Ahmad A. Formulation and penetration testing of ethosome azelaic acid on abdomen skin white rats (Rattus norvegicus) with franz diffusion cell. Int Pharm Clin Res 2018;11:327-30.

12. Dhamecha DL, Rathi AA, Saifee M, Lahoti SR, Dehghan MH. Drug vehicle based approaches of penetration enhancement. Int J Pharm Pharm Sci 2009;1:24-46. 